\title{
Sentinels in the visual system
}

\author{
Marco Tamietto ${ }^{1}$ and Beatrice de Gelder ${ }^{1,2 *}$ \\ 1 Cognitive and Affective Neuroscience Laboratory, Tilburg University, Tilburg, Netherlands \\ ${ }_{2}$ Martinos Center for Biomedical Imaging, Massachusetts General Hospital and Harvard Medical School, Boston, MA, USA \\ ${ }^{*}$ Correspondence: degelder@nmr.mgh.harvard.edu
}

\section{A commentary on}

Blindsight depends on the lateral geniculate nucleus

by Schmid, M. C., Mrowka, S. W., Turchi, J., Saunders, R. C., Wilke, M., Peters, A. J., Ye, F. Q., and Leopold, D. A. (2010) Nature 466, 373-377.

The human visual system, like that of other mammals, comprises a multiplicity of parallel visual pathways of old evolutionary origin that bypass the primary visual cortex (V1; Milner and Goodale, 2006; Tamietto and de Gelder, 2010). We currently know very little about these V1-independent pathways, but it makes sense from an evolutionary standpoint that these pathways are not simply vestigial, but may continue to be active and perform some visual functions even when V1 is damaged or denervated. To quote W. James "the main function [of such V1-independent systems] is that of sentinels which when beams of light move over then, cry, 'Who goes there' and calls the fovea to the spot" (James, 1893).

A recent study by Schmid et al. (2010) published in Nature highlights the contribution of the lateral geniculate nucleus (LGN) in V1-independent vision in monkeys. They show that macaque monkeys with permanent $\mathrm{V} 1$ lesions are able to locate correctly high-contrast stimuli presented in the portion of the visual field affected by the lesion (scotoma). Critically, however, reversible inactivation of the LGN in the lesioned hemisphere abolishes behavioral detection and the associated fMRI responses in extrastriate visual cortex.

What new light do these important animal findings throw on the understanding of homologous systems in humans and, more broadly, on the neural underpinnings of visual awareness?

A first issue concerns the putative critical role of LGN in sustaining all forms $\mathrm{V} 1$-independent residual vision. In recent years, a broad spectrum of visual abilities persisting in humans after V1 lesions has been documented (blindsight; Weiskrantz, 2010). These include target detection, visually guided behavior, and the capacity to judge stimulus properties such as shape, color, motion direction, facial expression, and body postures. Given the existence of multiple V1-independent pathways, some of which do not involve LGN (Lyon et al., 2010), it makes sense to expect that these different abilities rely on different V1-independent pathways that are partially segregated starting already from early subcortical sites (Danckert and Rossetti, 2005). In fact, a recent study on a patient with V1 lesion and blindsight followed the same logic of the study by Schmid and colleagues, but selectively knocked-out the superior colliculus (SC) instead of the LGN (Tamietto et al., 2010). Results showed that behavioral (i.e., visually guided manual responses) as well as fMRI evidence of V1-independent visual processing disappear when stimuli that are invisible to the SC, but normally visible to the LGN, are presented. Therefore, it remains an open issue to understand which residual visual functions draw on the critical contribution of LGN, and which are based on other V1-independent pathways encompassing the SC and/or the pulvinar.

A directly related matter is the "causal role [of the LGN] in V1-independent processing of visual information" (Schmid et al., 2010). At least two alternative possibilities are consistent with evidence that inactivation of the LGN abolishes behavioral evidence of visual processing and fMRI responses in extrastriate areas. At one extreme, LGN is the (first or most critical) site where visual computations responsible for the instances of blindsight studied are made (e.g., motion, detection, etc.), and these LGN computations are those that critically impact on brain structures linked to behavioral outputs. At the other extreme, LGN may not contribute any critical visual processing in the absence of $\mathrm{V} 1$, but simply operates as a relay station that enables other subcortical structures (e.g., the SC) to interact with extrastriate cortices. In the latter case, this interaction via LGN is critical for behavioral evidence of V1-independent visual processing, and LGN would thus perform a relay function previously attributed to some extent to the pulvinar. Direct testing of these two possibilities, for instance by alternatively inactivating in the same experiment the LGN but not the SC and vice versa, will be important to clarify the nature of the contribution of various subcortical structures in V1-independent vision.

Thirdly, V1-independent vision in humans and monkeys has been demonstrated in hemispherectomized subjects, in whom the entire cortical mantel of one hemisphere has been removed (Ptito et al., 1996; Tomaiuolo et al., 1997; Ptito and Leh, 2007; Leh et al., 2010). This implies that, at least in some cases, the contribution of the cortex may not be necessary for blindsight to occur, and attention must be paid to subcortical structures capable to perform visual analysis and influence behavior in the absence of any cortical contribution. The independency of spared visual abilities from any cortical function may be due to considerable neural plasticity occurring over time and enabling subcortical structures to take over visual functions normally performed by the cortex. Such neural reorganization is not at stake in the results by Schmid et al., as monkeys were tested shortly after the V1 lesion, but it will be important for future research to assess the timing and amount of neural reorganization, especially at the subcortical level, in V1-independent vision.

Finally, blindsight in humans involves two components: clinical blindness ensuing from V1 damage (the "blind" component of blindsight), and the retained ability to respond to stimuli without visual awareness (the "sight" component of the phenomenon; Sanders et al., 1974). Frequently, however, these two components do not co-occur and blindsight cannot be claimed. For instance, 
several patients who show visually guided behavior after $\mathrm{V} 1$ lesions also retain some degree of visual awareness for the stimuli to which they respond, a condition that has been termed Riddoch syndrome (Zeki and Ffytche, 1998). Animal research is also relevant for a better insight into the role of striate and extrastriate cortex in mediating consciousness. In fact, previous demonstrations of blindsight in animals established both components of the phenomenon; i.e., that monkeys with $\mathrm{V} 1$ lesions were able to detect and localize the stimulus presented in the scotoma, but also that in a forcedchoice procedure accompanying the main testing they categorized these trials as having "no stimulus" Cowey and Stoerig, 1995. In the study by Schmid et al., however, we do not know whether the stimuli presented in the scotoma were associated to some form of (degraded) visual awareness, and therefore we cannot conclude whether their monkeys with V1 lesions represented an animal model of human blindsight or of Riddoch syndrome. Besides that, it is an important and open question whether all
V1-independent visual abilities are to the same extent associated with consciousness or the absence thereof.

\section{REFERENCES}

Cowey, A., and Stoerig, P. (1995). Blindsight in monkeys. Nature 373, 247-249.

Danckert, J., and Rossetti, Y. (2005). Blindsight in action: what can the different sub-types of blindsight tell us about the control of visually guided actions? Neurosci. Biobehav. Rev. 29, 1035-1046.

James, W. (1893). Psychology. New York: Henry Holt.

Leh, S. E., Ptito, A., Schonwiesner, M., Chakravarty, M M., and Mullen, K. T. (2010). Blindsight mediated by an S-cone-independent collicular pathway: an fMRI study in hemispherectomized subjects. J. Cogn. Neurosci. 22, 670-682.

Lyon, D. C., Nassi, J. J., and Callaway, E. M. (2010). A disynaptic relay from superior colliculus to dorsal stream visual cortex in macaque monkey. Neuron 65, 270-279.

Milner,A.D., and Goodale, M.A. (2006). The Visual Brain in Action, 2nd Edn. Oxford: Oxford University Press.

Ptito, A., and Leh, S. E. (2007). Neural substrates of blindsight after hemispherectomy. Neuroscientist 13, 506-518.

Ptito, M., Herbin, M., Boire, D., and Ptito, A. (1996). Neural bases of residual vision in hemicorticectomized monkeys. Prog. Brain Res. 112, 385-404.

Sanders, M. D., Warrington, E. K., Marshall, J., and Weiskrantz, L. (1974). "Blindsight": vision in a field defect. Lancet 707-708.
Schmid, M. C., Mrowka, S. W., Turchi, J., Saunders, R. C., Wilke, M., Peters, A. J., Ye, F. Q., and Leopold, D. A. (2010). Blindsight depends on the lateral geniculate nucleus. Nature 466, 373-377.

Tamietto, M., Cauda, F., Corazzini, L. L., Savazzi, S., Marzi, C. A., Goebel, R., Weiskrantz, L., and de Gelder, B. (2010). Collicular vision guides nonconscious behavior. J. Cogn. Neurosci. 22, 888-902.

Tamietto, M., and de Gelder, B. (2010). Neural bases of the non-conscious perception of emotional signals. Nat. Rev. Neurosci. 11, 697-709.

Tomaiuolo, F., Ptito, M., Marzi, C. A., Paus, T., and Ptito, A. (1997). Blindsight in hemispherectomized patients as revealed by spatial summation across the vertical meridian. Brain 120, 795-803.

Weiskrantz,L. (2010). Blindsight in hindsight. Psychologists 23, 356-358.

Zeki, S., and Ffytche, D. H. (1998). The Riddoch syndrome: insights into the neurobiology of conscious vision. Brain 121, 25-45.

Received: 21 January 2011; accepted: 08 February 2011; published online: 22 February 2011.

Citation: Tamietto M and de Gelder B (2011) Sentinels in the visual system. Front. Behav. Neurosci. 5:6. doi: 10.3389/ fnbeh.2011.00006

Copyright $\odot 2011$ Tamietto and de Gelder. This is an openaccess article subject to an exclusive license agreement between the authors and Frontiers Media SA, which permits unrestricted use, distribution, and reproduction in any medium, provided the original authors and source are credited. 